\title{
Analysis of pemetrexed-based chemotherapy in the treatment of advanced colorectal cancer
}

\author{
Zhengyi Yu ${ }^{1 \#}$, Jiawei Wang ${ }^{1 \#}$, Xiaomin Cai ${ }^{1}$, Zhenzhen Gao ${ }^{1}$, Sailan Wang ${ }^{2}$, Yanhong Gu ${ }^{1}$ \\ ${ }^{1}$ Department of Oncology, the First Affiliated Hospital of Nanjing Medical University, Nanjing, China; ${ }^{2}$ Department of Obstetrics and Gynecology, \\ the First Affiliated Hospital of Nanjing Medical University, Nanjing, China \\ Contributions: Conception and design: Z Yu; Administrative support: Y Gu; Provision of study materials or patients: Z Yu, J Wang; (IV) Collection \\ and assembly of data: Z Yu, J Wang, X Cai; (V) Data analysis and interpretation: Z Yu, S Wang; (VI) Manuscript writing: All authors; (VII) Final \\ approval of manuscript: All authors. \\ "These authors contributed equally to this work. \\ Correspondence to: Yanhong Gu, MD, PhD. Department of Oncology, the First Affiliated Hospital of Nanjing Medical University, 300 Guangzhou Rd, \\ Nanjing, China. Email: guyhphd@163.com.
}

Background: In this study, we evaluated the therapeutic efficacy and toxicity profile of chemotherapy combinations containing pemetrexed for patients with metastatic colorectal cancer. We investigated the optimal chemotherapy treatment regimen to provide a new option for third-line or after treatment of patients with advanced colorectal cancer.

Methods: A total of 88 eligible patients with metastatic colorectal cancer were included in this study from April 2009 to March 2019 at the Department of Oncology, the First Affiliated Hospital of Nanjing Medical University. The baseline information and treatment outcomes of the patients were collected. Statistical analyses of different chemotherapy regimens focusing on objective response rate (ORR), disease control rate (DCR), progression-free survival (PFS), overall survival (OS), and toxicity were conducted. The superior treatment regimen was determined, and its clinical outcomes were compared with those of the other treatment combinations to explore the factors that potentially contributed to the curative effect.

Results: The 88 patients in this study received 18 treatment regimens. In total, 53 patients had progressive disease (PD), 34 patients had stable disease (SD), 1 patient was assessed as complete response (CR), and no patients had a partial response (PR). The optimal regimen was pemetrexed + S-1 + bevacizumab. The 21 patients treated with this regimen exhibited a higher DCR [61.90\% vs. 32.84\%, odds ratio (OR) $=3.324$; 95\% confidence interval (CI): 1.201-9.196, $\mathrm{P}=0.018$ ] than patients treated with the other chemotherapy regimens. Moreover, the median PFS of this regimen was 4.57 (2.62-6.51) months, which was significantly longer [hazard ratio $(\mathrm{HR})=0.566$; 95\% CI: 0.330-0.971, P=0.039] than the $2.57(2.18-2.95)$ months of the other regimens. In terms of toxicity, leukopenia (34.1\%) and neutropenia (34.1\%) had the highest incidence of all-grade adverse events (AEs). Grade 3-4 AEs included neutropenia (15.9\%), leukopenia (11.4\%), thrombocytopenia $(2.3 \%)$, and anemia $(1.1 \%)$.

Conclusions: The combination of pemetrexed $+\mathrm{S}-1+$ bevacizumab was found to be the optimal treatment regimen. This combination was superior to the other treatment regimens in terms of DCR and PFS with controllable toxicity. These results warrant further prospective exploratory clinical trials for pemetrexedbased chemotherapy in metastatic colorectal cancer.

Keywords: Metastatic colorectal cancer; advanced colorectal cancer; pemetrexed; third-line treatment

Submitted Jan 29, 2020. Accepted for publication Sep 18, 2020.

doi: 10.21037/atm-20-1095

View this article at: http://dx.doi.org/10.21037/atm-20-1095

(c) Annals of Translational Medicine. All rights reserved. 


\section{Introduction}

The worldwide incidence and disease burden of colorectal cancer remain high. In the United States, there were approximately 1.8 million new cases of colorectal cancer in 2018, ranking this disease third in the overall tumor incidence rate (1). In China, the incidence of colorectal cancer is approximately $28.20 / 100,000$, with a male to female ratio of 1.32 , and the mortality rate is approximately $13.61 / 100,000$, with a male to female ratio of 1.34 . The incidence of colorectal cancer in China is higher in urban areas than in rural areas $(33.51 / 100,000$ vs. $21.41 / 100,000)$. In terms of regional distribution, the eastern region of China has a higher incidence of colorectal cancer. Furthermore, a considerable number of patients with colorectal cancer are already at an advanced stage of the disease at the time of diagnosis (2).

At present, the National Comprehensive Cancer Network (NCCN) guidelines recommend oxaliplatin, irinotecan, and fluorouracil as the first-line and secondline drug treatments for metastatic colorectal cancer. The targeted drugs that can be used include cetuximab or panitumumab (recommended for patients with wildtype RAS and BRAF genes), bevacizumab, aflibercept, and ramuciruma. As a third-line treatment, only cetuximab + irinotecan (recommended for patients with wild-type RAS and BRAF genes) + vimofenib/trametinib/dabrafenib/ binimetinib (recommended for patients with BRAF gene mutations), regorafenib, or TAS-102 is recommended. With the development of immunotherapy, an immunotherapeutic intervention can be applied at any stage of treatment for patients with microsatellite instability-high (MSI-H)/ deficient-mismatch repair (d-MMR) tumors (3). Moreover, in recent clinical practice, regorafenib plus nivolumab has become a novel treatment combination for microsatellite stable (MSS) colorectal cancer (4). As the treatment of colorectal cancer gains more research attention, more genomic signatures are being discovered. Patients with human epidermal growth factor receptor 2 (HER2)amplified colorectal cancer, for instance, were found to be sensitive to HER2-targeted therapy with pertuzumab plus trastuzumab (5), and it may be possible to use tyrosine kinase inhibitors (TKIs) to treat patients with NTRK gene fusions (6). The Chinese Society of Clinical Oncology (CSCO) guidelines also include raltitrexed and fruquintinib as alternative third-line drug treatments.

Pemetrexed is a multi-target antifolate preparation with a pyrimidine group at the core. It destroys the cell's folate-dependent metabolic processes and inhibits cell replication, thereby inhibiting tumor growth (7). The drug is long-used in the treatment of non-small cell lung cancer, and has demonstrated good synergy with platinum, antiangiogenesis drugs and immunotherapy drugs. Pemetrexed also has low therapeutic toxicity, and the main adverse reactions include decreased white blood cells (8). As a classic antimetabolite, pemetrexed was also demonstrated by Zhang et al. to have effective antitumor activity in in vitro experiments of colorectal cancer (9). In a phase II clinical trial published in 2007, pemetrexed was used in combination with irinotecan versus FOLFIRI for first-line treatment of metastatic colorectal cancer but did not achieve results that exceeded those of the standard treatments (10). A Chinese study used pemetrexed combined with oxaliplatin, irinotecan, or cisplatin as a third-line treatment for metastatic colorectal cancer. The main endpoint was progressionfree survival (PFS), which reached 2.5 months (11). In the outcomes of a phase II clinical trial, the median progression-free survival (mPFS) of pemetrexed combined with gemcitabine as a third-line treatment for metastatic colorectal cancer was 2.1 months. The preclinical data made by Passardi et al. also indicated the curative effect of pemetrexed and gemcitabine. However, the later design was more susceptible to the effect of gemcitabine, because the dose of pemetrexed was relatively low $\left(150 \mathrm{mg} / \mathrm{m}^{2} / 14\right.$ days) (12). Another attempt to use pemetrexed monotherapy as a thirdline treatment achieved an mPFS of 1.6 months. Although the PFS was not very long, 7 patients achieved stable disease for more than 6 months, which suggested that certain patients could benefit from pemetrexed monotherapy (13). Zhang et al. used second-line or third-line treatment regimens containing pemetrexed to treat 17 patients with metastatic colorectal cancer, resulting in an mPFS of 2.0 months (14). Regorafenib, the standard third-line treatment for metastatic colorectal cancer, had an mPFS of 3.2 months in the Asian population of the CONCUR study (15), while the mPFS of fruquintinib in the FRESCO study was 3.7 months (16). Compared with standard treatments, pemetrexed-based treatment has not shown obvious advantages, but given that the combinations of pemetrexed-based treatment are not limited to the above types, we believe that the drug still has potential in the treatment of colorectal cancer.

After a comprehensive update of the treatment guidelines and the standardization of clinical practice, the survival time of patients with advanced colorectal cancer has been substantially extended, and more patients are eligible for 
third-line and later treatments. In view of the lack of thirdline treatment options for metastatic colorectal cancer, our study aimed to retrospectively assess the effectiveness of pemetrexedbased chemotherapy as a third-line or further treatment in patients with metastatic colorectal cancer, and to provide new therapeutic options for such patients by determining the optimal treatment regimen. We present the following article in accordance with the STROBE reporting checklist (available at http://dx.doi.org/10.21037/atm-20-1095).

\section{Methods}

The study was conducted in accordance with the Declaration of Helsinki (as revised in 2013). The study was approved by the Ethics Committee of the First Affiliated Hospital of Nanjing Medical University (2019-SR-301).

Patients with colorectal cancer who had been treated in the Oncology Department of the First Affiliated Hospital of Nanjing Medical University from April 2009 to March 2019 were selected. A unified form was used to collect the following information for each patient: name, hospital number, sex, age, diagnosis, disease process, location of the primary tumor, genetic mutations (RAS, BRAF), prior use of targeted drugs, regimens, objective response rate (ORR), disease control rate (DCR), PFS, overall survival (OS), and toxicity.

\section{Inclusion criteria}

The inclusion criteria for patients in this study were as follows: (I) treated in the Oncology Department of the First Affiliated Hospital of Nanjing Medical University from April 2009 to March 2019 and diagnosed with colorectal cancer by colonoscopy or surgery; (II) metastasis judged by clear imaging or pathology; (III) had previously received standard chemotherapy (fluorouracil, irinotecan, and oxaliplatin, according to the standard chemotherapy regimen) and experienced treatment failure, with treatment failure being defined as having clear evidence of imaging or clinical progression during the treatment or within 3 months after the last treatment, or withdrawal from standard treatment due to intolerance of chemotherapy adverse events; (IV) chemotherapy based on pemetrexed used as the third-line or further treatment, with the dose of pemetrexed being $500 \mathrm{mg} / \mathrm{m}^{2} / 21$ days, and with doses of other drugs being administered according to instructions; $(\mathrm{V})$ at least one follow-up within 3 months after the target regimen, with the interval between each evaluation not exceeding 3 months; (VI) at least 6 months postchemotherapy with pemetrexed.

\section{Exclusion criteria}

The exclusion criteria for patients in this study were as follows: (I) presence of other malignant tumors; (II) patient performance status scores of 3 or higher recorded in the medical records during treatment; (III) inability to assess curative effects.

\section{Data collection and follow-up}

The keywords "colon cancer", "rectal cancer", "colon malignant tumor", "rectal malignant tumor", and "pemetrexed" were queried in the hospital information system, and were initially screened for analysis. The pemetrexed-based regimen is used to treat patients with advanced colorectal cancer. The eligibility of cases for inclusion was determined using the pathological and imaging examination systems further. All patients who met the criteria were included for analysis. Baseline information and treatment outcomes of patients were collected and analyzed. Therapeutic outcomes included ORR, DCR, PFS, OS, and toxicity. The therapeutic effect was assessed using the Response Evaluation Criteria in Solid Tumors (RECIST) 1.1, and toxicity was evaluated according to Common Terminology Criteria for Adverse Events (CTCAE) 4.0. Progression was judged by computed tomography (CT) scan or other imaging data. The followup ended on March 26, 2019. Survival data were collected by viewing medical records or telephone follow-up. OS was defined as the time between pemetrexed-based chemotherapy and death or last follow-up. If a patient was lost to follow-up, survival time was defined as the time of last visit. The most effective chemotherapy regimen and its toxicity profile were determined, and the clinical data of this regimen were compared with those of other regimens to explore the possible factors that influenced treatment response.

\section{Statistical analysis}

Statistical analysis was performed using the SPSS 24.0 (IBM SPSS Statistics, IBM Corporation, Armonk, NY, USA) software package. Mean \pm standard deviation was used to describe the distribution of quantitative data, and independent samples $t$ tests were used to compare 
Table 1 Patient demographic and baseline characteristics

\begin{tabular}{|c|c|}
\hline Baseline information & Result \\
\hline Number of patients & 88 \\
\hline \multicolumn{2}{|l|}{ Gender } \\
\hline Male & 54 \\
\hline Female & 34 \\
\hline \multicolumn{2}{|l|}{ Age (years) } \\
\hline Median & 60 \\
\hline Range & $36-79$ \\
\hline \multicolumn{2}{|l|}{ Disease process } \\
\hline Initial diagnosis stage IV & 38 \\
\hline Relapse stage IV & 50 \\
\hline \multicolumn{2}{|l|}{ Metastasis site } \\
\hline Abdominal cavity & 10 \\
\hline Liver & 58 \\
\hline Lung & 41 \\
\hline Lymph nodes & 22 \\
\hline Other & 17 \\
\hline \multicolumn{2}{|l|}{ Primary tumor location } \\
\hline Left colon & 62 \\
\hline Right colon & 26 \\
\hline \multicolumn{2}{|l|}{ RAS, BRAF gene mutation, $\mathrm{n}(\%)$} \\
\hline All wild type & $16(18.18)$ \\
\hline Unknown & $52(59.09)$ \\
\hline KRAS & $19(21.59)$ \\
\hline BRAF & $1(1.14)$ \\
\hline \multicolumn{2}{|c|}{ Targeted drug use in previous therapies, $\mathrm{n}(\%)$} \\
\hline Unused & $42(47.73)$ \\
\hline Bevacizumab & $34(38.64)$ \\
\hline Cetuximab & $9(10.23)$ \\
\hline Other & $3(3.41)$ \\
\hline \multicolumn{2}{|c|}{ Compatibilities of chemotherapy with pemetrexed, $\mathrm{n}(\%)$} \\
\hline Pemetrexed + S-1 & $26(29.54)$ \\
\hline Pemetrexed + S-1 + Bevacizumab & $21(23.86)$ \\
\hline Pemetrexed + Capecitabine & $7(7.95)$ \\
\hline Pemetrexed & $6(6.82)$ \\
\hline Pemetrexed + Oxaliplatin & $5(5.68)$ \\
\hline Pemetrexed + Bevacizumab & $5(5.68)$ \\
\hline Other compatibility regimens & $18(20.45)$ \\
\hline
\end{tabular}

differences between groups. Frequency and composition ratios were used to describe the distribution of qualitative data. Differences between groups were analyzed using the chi-square test or Fisher's exact probability method. Binary logistic regression analysis was used to compare outcome ratios of different treatment options. The Kaplan-Meier method was used for survival analysis and to plot survival curves, and the log-rank method was used to compare survival differences between groups. All tests were two sided, and a $P$ value $<0.05$ was considered statistically significant. The distribution chart of the start time of treatment, the waterfall chart of the changing rate of the target lesions, and the changing rate of carcinoembryonic antigen (CEA) were depicted using Origin 2018 software.

\section{Results}

\section{Patient characteristics}

Ninety-five patients with colorectal cancer had been treated with pemetrexed in the hospital and were potentially eligible for inclusion. Five of these patients were lost to follow-up within 3 months, and two were excluded as the curative effect could not be assessed. Eventually, 88 patients were included in the analysis. The demographic and baseline characteristics of the 88 evaluable patients are summarized in Table 1 . The cohort comprised 54 males and 34 females with a median age of 60 (range, 3679) years. The treatment regimens used included pemetrexed + S-1 (26 cases), pemetrexed + S-1 + bevacizumab (21 cases), pemetrexed + capecitabine ( 7 cases), pemetrexed monotherapy (6 cases), pemetrexed + oxaliplatin ( 5 cases), pemetrexed + bevacizumab (5 cases), pemetrexed + cetuximab (4 cases), pemetrexed + gemcitabine ( 3 cases), pemetrexed + capecitabine + bevacizumab (2 cases), pemetrexed + oxaliplatin + apatinib (1 case), pemetrexed + capecitabine + apatinib (1 case), pemetrexed + nedaplatin + cetuximab ( 1 case), pemetrexed + capecitabine + cetuximab ( 1 case), pemetrexed + raltitrexed $(1$ case), pemetrexed + raltitrexed + bevacizumab (1 case), pemetrexed + albumin paclitaxel (1 case), pemetrexed + irinotecan (1 case), and pemetrexed + nedaplatin (1 case). In total, the patients in this cohort had received 18 treatment regimens.

\section{Therapeutic efficacy, toxicity, and selection of the most effective regimen}

From the patient pool, 1 patient was evaluated as complete 
Table 2 Comparison of the therapeutic effect between pemetrexed $+\mathrm{S}-1+$ bevacizumab and other regimens

\begin{tabular}{|c|c|c|c|c|c|}
\hline Treatment outcomes & Other regimens & $\mathrm{Pem}+\mathrm{S}-1+\mathrm{Bev}$ & OR $(95 \% \mathrm{Cl})$ & $\mathrm{HR}(95 \% \mathrm{Cl})$ & $\mathrm{P}$ \\
\hline CR & 1 & 0 & - & - & - \\
\hline PR & 0 & 0 & - & - & - \\
\hline SD & 21 & 13 & - & - & - \\
\hline DCR (\%) & 32.84 & 61.90 & $3.324(1.201-9.196)$ & - & $0.018^{*}$ \\
\hline Events of progression or death & 65 & 17 & - & - & - \\
\hline mPFS (m) & $2.57(2.18-2.95)$ & $4.57(2.62-6.51)$ & - & $0.566(0.330-0.971)$ & $0.039^{\star}$ \\
\hline Events of death & 41 & 13 & - & - & - \\
\hline
\end{tabular}

*, P<0.05. CR, complete response; PR, partial response; SD, stable disease; PD, progressive disease; DCR, disease control rate; mPFS, median progression-free survival; mOS, median overall survival; OR, odds ratio; HR, hazard ratio; Pem, Pemetrexed; Bev, Bevacizumab; $\mathrm{Cl}$, confidence interval.

A

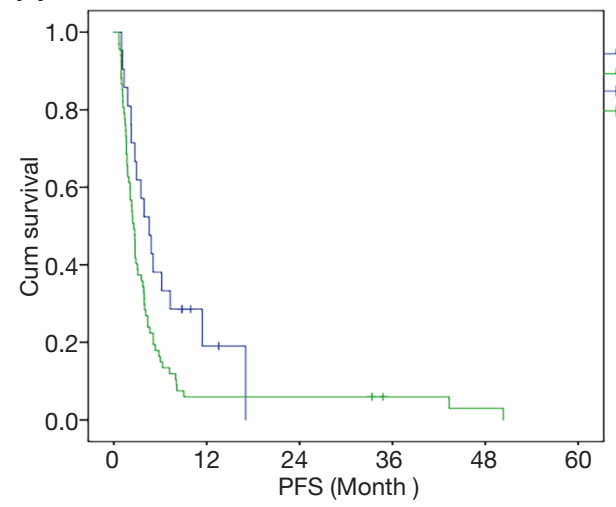

B

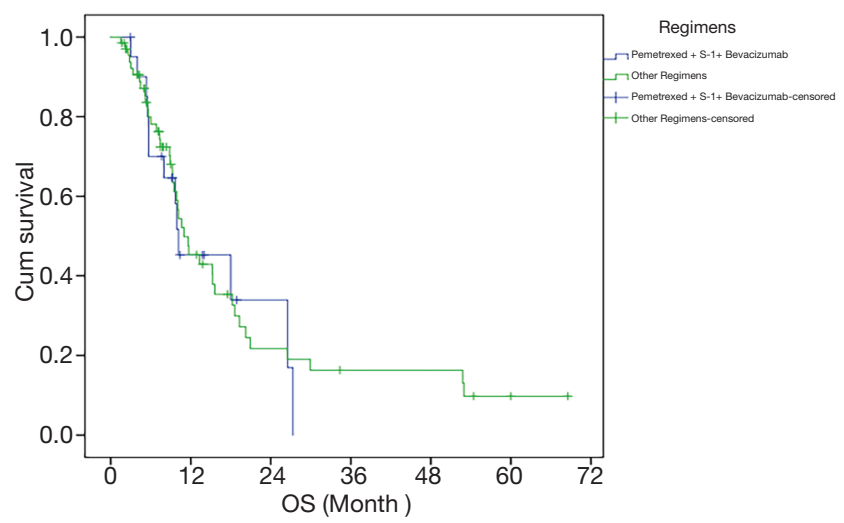

Figure 1 Kaplan-Meier curves depicting survival in patients with different treatment combinations. (A) Kaplan-Meier curve for progression-free survival; (B) Kaplan-Meier curve for overall survival. PFS, progression-free survival; OS, overall survival. response (CR), no patients had partial response (PR), 34 patients had stable disease (SD), and 53 patients had progressive disease (PD). The 88 patients had a median follow-up of 8.85 months. The ORR of all pemetrexedcontaining chemotherapy regimens was $1.14 \%(1 / 88)$, the DCR was $39.77 \%(35 / 88)$, the mPFS was 2.73 (2.18-3.28) months, and the median overall survival (mOS) was 11.00 (7.69-14.31) months. For treatment regimens that treated more than 20 patients, a preliminary statistical analysis showed that the DCR of the pemetrexed $+\mathrm{S}-1$ regimen was $42.31 \%(11 / 26)$, and the mPFS was 2.8 months, while the DCR of pemetrexed $+\mathrm{S}-1+$ bevacizumab was $61.90 \%(13 / 21)$, and the mPFS was 4.57 months. The DCR of the other 41 patients treated by the rest 16 regimens was $26.19 \%(11 / 42)$, and the mPFS was 2.4 months. Therefore, pemetrexed $+\mathrm{S}-1+$ bevacizumab was confirmed as the superior regimen.

Next, the efficacy of pemetrexed $+\mathrm{S}-1+$ bevacizumab was compared with that of the other regimens. The results are shown in Table 2 and Figure 1. The median follow-up time with pemetrexed $+\mathrm{S}-1+$ bevacizumab was 9.23 months, compared to 8.33 months with the other regimens. Pemetrexed $+\mathrm{S}-1+$ bevacizumab had a higher DCR than the other regimens $[61.90 \%$ vs. $32.84 \%$, $\mathrm{OR}=3.324,95 \%$ confidence interval $(\mathrm{CI})=1.201-9.196$, $\mathrm{P}=0.018]$, and an mPFS of 4.57 (2.62-6.51) months, compared with the $2.57(2.18-2.95)$ months of the other schemes $(\mathrm{HR}=0.566,95 \% \mathrm{CI}=0.33-0.971, \mathrm{P}=0.039)$. 

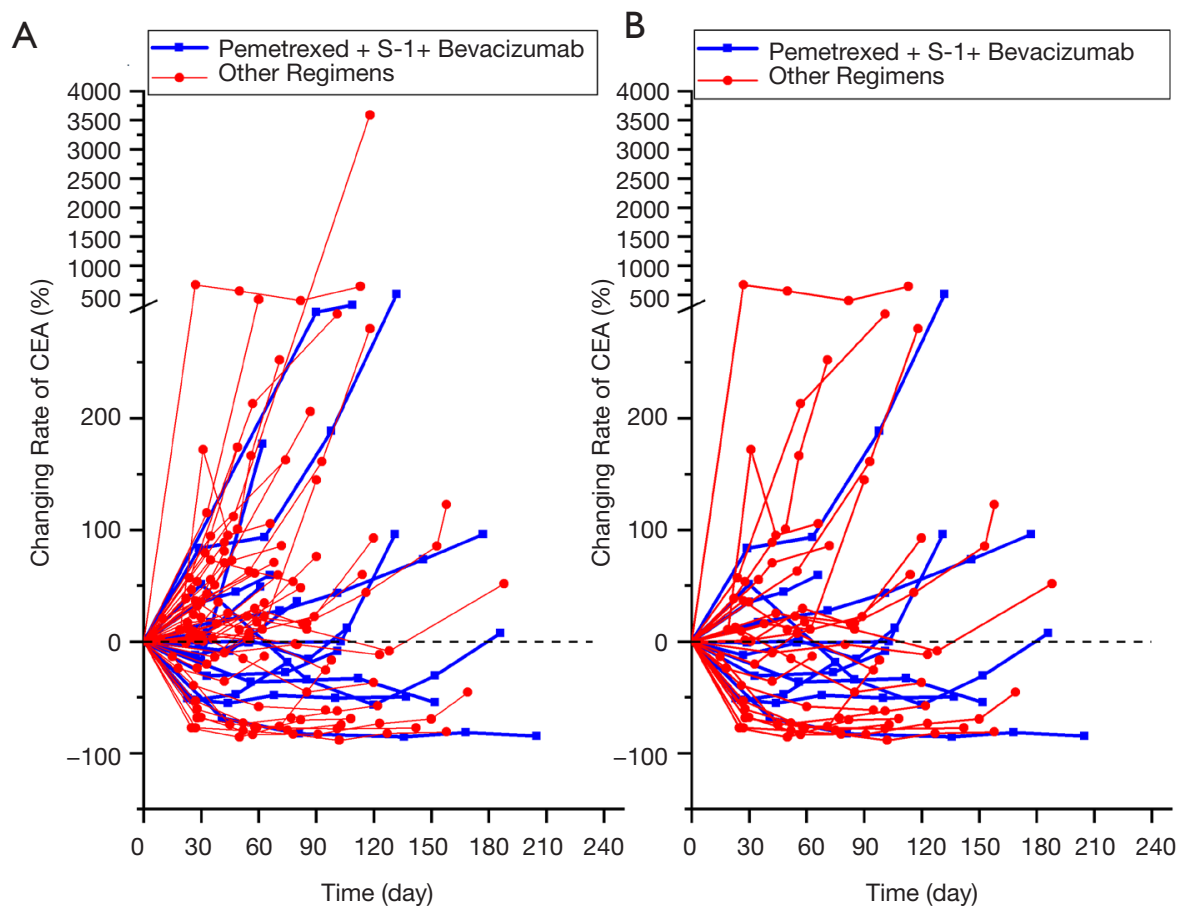

Figure 2 The changing rate of CEA during treatment of CEA-positive ( $>4.71 \mathrm{ng} / \mathrm{mL}$ ) patients in the two groups. (A) All positive patients, (B) patients excluding progression within 2 months. CEA, carcinoembryonic antigen.

The changing levels of CEA during the treatment of CEA-positive $(>4.71 \mathrm{ng} / \mathrm{mL})$ patients using the superior regimen and other regimens are shown in Figure 2, and the changing rate of target lesions in patients is shown in Figure 3. The ORRs of the two groups were 0 and $1.14 \%$, respectively. The single patient evaluated as CR might have been a coincidence. The estimated mOS of pemetrexed + S-1 + bevacizumab was 10.17 (2.06-18.27) vs. 11.00 (7.61-14.39) months (HR $=1.095$, 95\% CI: 0.582-2.061, $\mathrm{P}=0.777$ ) with the other regimens, with no significant difference.

In terms of toxicity, leukopenia (34.1\%) and neutropenia $(34.1 \%)$ were the most common all-grade adverse events. Elevated aspartate aminotransferase (22.7\%), anemia $(21.6 \%)$, thrombocytopenia $(18.2 \%)$, alanine aminotransferase $(13.6 \%)$, and proteinuria (11.4\%) also had a high incidence. Grade 3-4 adverse events included leukopenia (11.4\%), neutropenia (15.9\%), thrombocytopenia (2.3\%), and anemia (1.1\%); 2 cases of neutropenia were grade 4 and occurred in the other regimen groups. The rates of all-grade leukopenia $(52.4 \%$ vs. $28.4 \%$, $\mathrm{P}=0.043$ ), neutropenia (52.4\% vs. $28.4 \%, \mathrm{P}=0.043$ ), and proteinuria $(28.6 \%$ vs. $6.0 \%, \mathrm{P}=0.014)$ were higher among patients who received pemetrexed $+\mathrm{S}-1+$ bevacizumab than among patients treated with the other regimens. However, there were no significant differences in the incidence of grade 3-4 adverse events between the two groups (Table 3).

\section{Differences in clinical characteristics of patients between the superior regimen and other regimens}

In view of the superiority of pemetrexed + S- $1+$ bevacizumab compared with other treatments, the clinical characteristics of the two groups of patients were analyzed. The results are shown in Table 4. The proportion of patients with combined lung metastasis in the superior regimen group was lower than those in the other regimens group $(14.29 \%$ vs. $56.72 \%, \mathrm{P}=0.001)$, and the proportion of patients who had used bevacizumab as a previous treatment was higher ( $66.67 \%$ vs. $29.85 \%, \mathrm{P}=0.002)$, while the proportion of patients who did not use any targeted drugs was lower $(14.29 \%$ vs. $58.21 \%, \mathrm{P}<0.001)$. Also, the difference in the composition of common gene mutations between the two groups of patients was statistically significant $(\mathrm{P}=0.002)$. The KRAS gene mutation rate in the pemetrexed + S-1 + bevacizumab group was higher than 


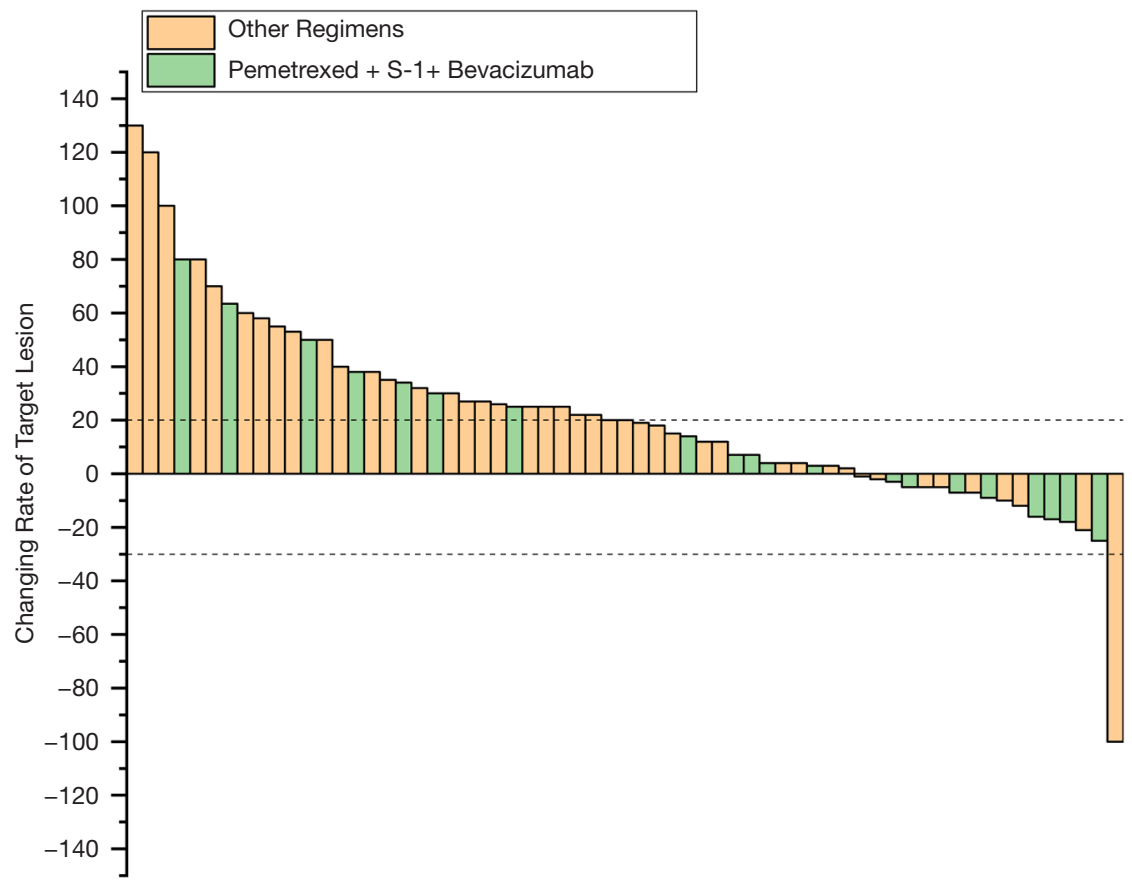

Figure 3 Changing rate of target lesions of patients in the two groups. Changing rate was evaluated when the curative effect or progression appeared.

that in patients treated with the other regimens $(42.86 \%$ vs. $21.59 \%)$. The proportion of unknown mutation statuses in the pemetrexed $+\mathrm{S}-1+$ bevacizumab group was lower (28.57\% vs. $59.09 \%)$. Finally, the time distribution of treatment initiation in the two groups was different, and the superior treatment group was closer overall (Figure 4).

\section{Discussion}

A number of factors have played an important role in the development of treatment options for metastatic colorectal cancer. These factors include the rise of multi-disciplinary treatment (MDT), the compatibility of chemotherapy drugs and schedule changes, the development of refined patient classification and conversion therapies, and the application of targeted drugs and immunotherapy. As early as the 2004 AVF2107g study, the mOS of patients treated with first-line chemotherapy combined with bevacizumab was 20.3 months (17). In the CALGB80405 study, the mOS of KRAS wild-type patients in the cetuximab group was 30.0 months, compared with 29.0 months in the bevacizumab group (18). The current survival period of colorectal cancer patients is evidently longer than before, and the possibilities and the demand of patients receiving further treatment are gradually increasing. Therefore, it is of practical significance to explore third-line and further treatments for colorectal cancer.

This study analyzed 88 patients who used pemetrexedbased regimens as a third-line or further treatment. The mPFS was 2.73 months; however, the data demonstrated no equivalent advantage in PFS compared with the standard treatment. In contrast, patients treated with pemetrexed + S-1 + bevacizumab achieved a greater therapeutic effect. Although the number of cases in this treatment group was small, the difference was still statistically significant. Pemetrexed + S-1 + bevacizumab was demonstrated to have a greater advantage over other schemes in DCR and PFS. At the same time, this difference might have stemmed from accidental events, and further research involving a larger sample size may be required. In the estimated patient mOS data, although there was no significant difference between the two groups, the mOS reported was significantly longer than those of previous clinical trials. Further exploration of the patient treatment process found that most patients continued on to other treatment after pemetrexed-based chemotherapy, including local treatment (radiotherapy, hepatic arterial embolization, radiofrequency ablation, metastatic resection), reapplication of oxaliplatin-based 
Table 3 Toxicity according to CTCAE4.0

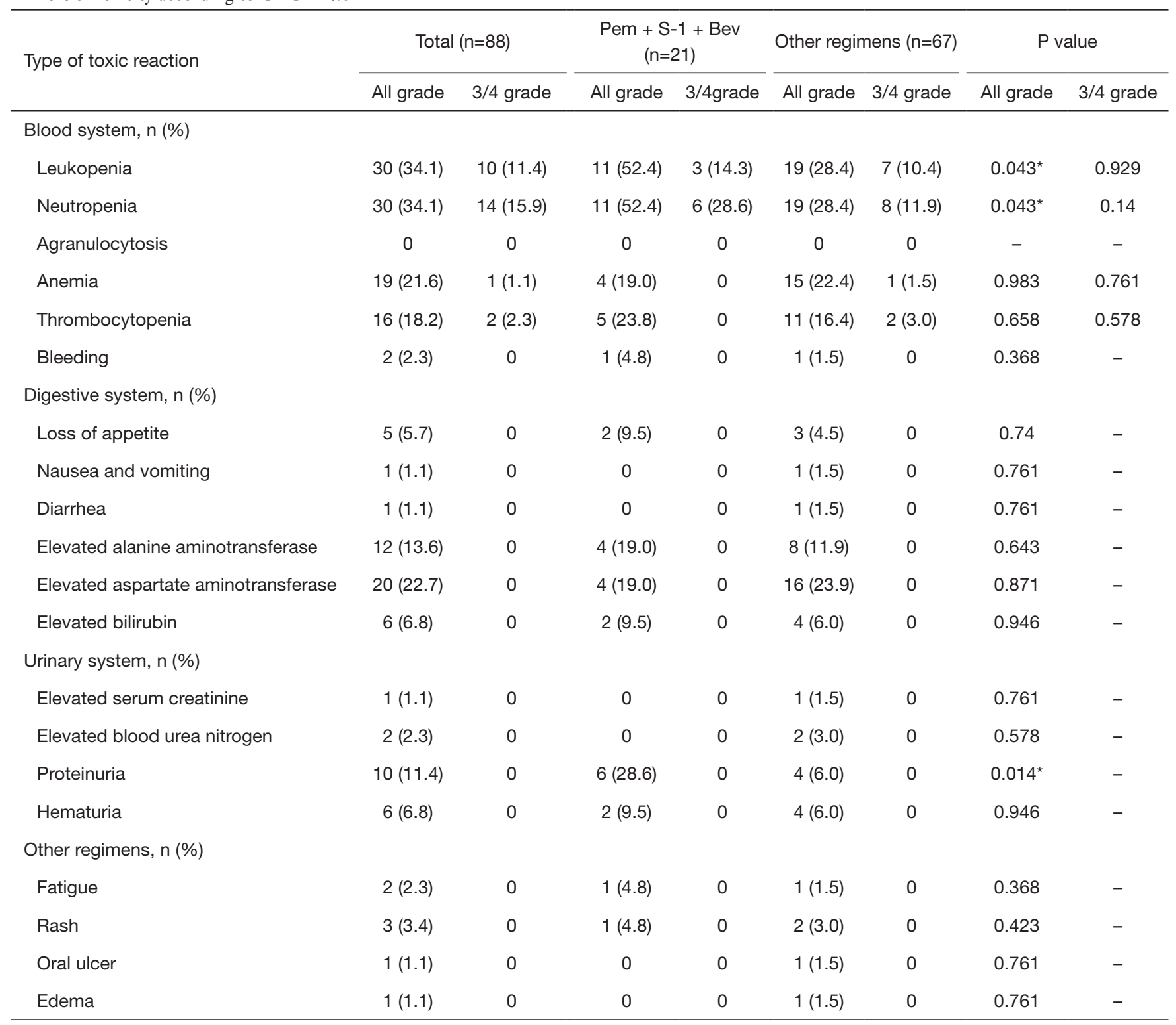

*, $\mathrm{P}<0.05$. Pem, Pemetrexed; Bev, Bevacizumab.

chemotherapy, or bevacizumab maintenance, or they participated in clinical trials (anlotinib, regorafenib). Based on the fact that patients agreed to use non-standard treatment, we speculate that the patients included in this study had a strong willingness for treatment and might have been in a favorable economic position; these factors can have a positive effect on patient prognosis. The opportunity to receive continuous treatment was also one of the factors influencing patient survival benefit, and the OS benefit in this population of patients might have been significantly prolonged due to factors beyond just the specific treatment plan.

In terms of adverse events, pemetrexed $+\mathrm{S}-1+$ bevacizumab was associated with more hematological toxicity, although the incidence of grade 3-4 toxicities was acceptable. Furthermore, these 88 patients were heavily treated. Some patients started treatment with long-term cumulative toxicity caused by the previous treatment. Additionally, patients with grade 3-4 hematological toxicities had also experienced hematological adverse events 
Table 4 Differences in clinical characteristics between pemetrexed + S-1 + bevacizumab and other regimens

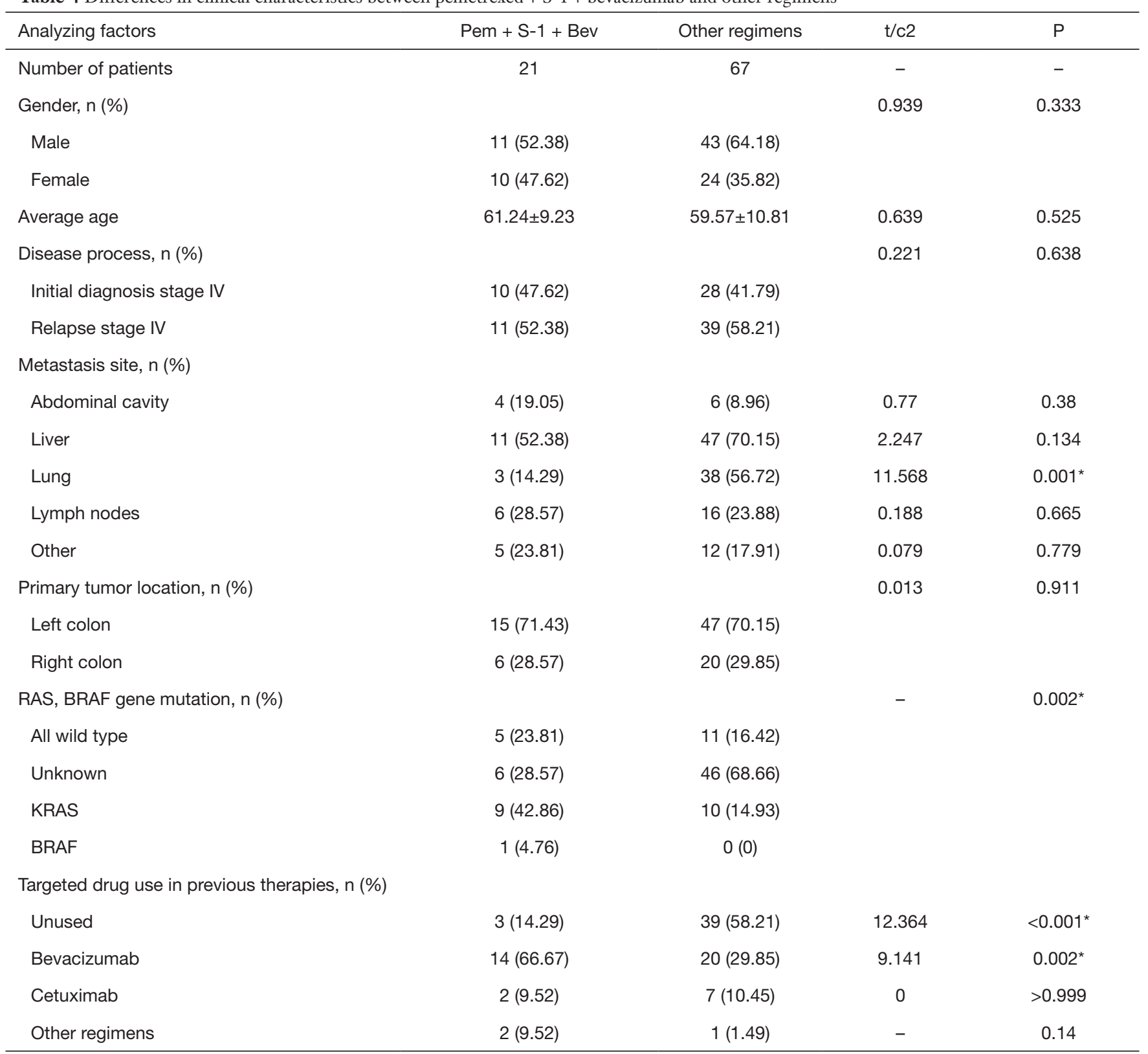

${ }^{*}, \mathrm{P}<0.05$. Pem, Pemetrexed; Bev, Bevacizumab.

during their previous treatment. Therefore, exact toxicity of pemetrexed $+\mathrm{S}-1+$ bevacizumab should be further explored in future prospective studies.

Pemetrexed, an antifolate agent, was found to have similar effects to raltitrexed and fluorouracil, which are recommended by the guidelines. The three pharmacological targets of these drugs include thymidylate synthase (TS, thymidylate synthase), but the specific mechanism of action varies. For example, pemetrexed combined with cisplatin showed similar therapeutic effects in malignant pleural mesothelioma to raltitrexed combined with cisplatin (19). As a classic chemotherapy drug, pemetrexed achieves significant results in non-small cell lung cancer and other malignancies. Therefore, we believe that pemetrexed has potential as a third-line treatment for colorectal cancer.

S-1, as a compound preparation, consists of tegafur and 


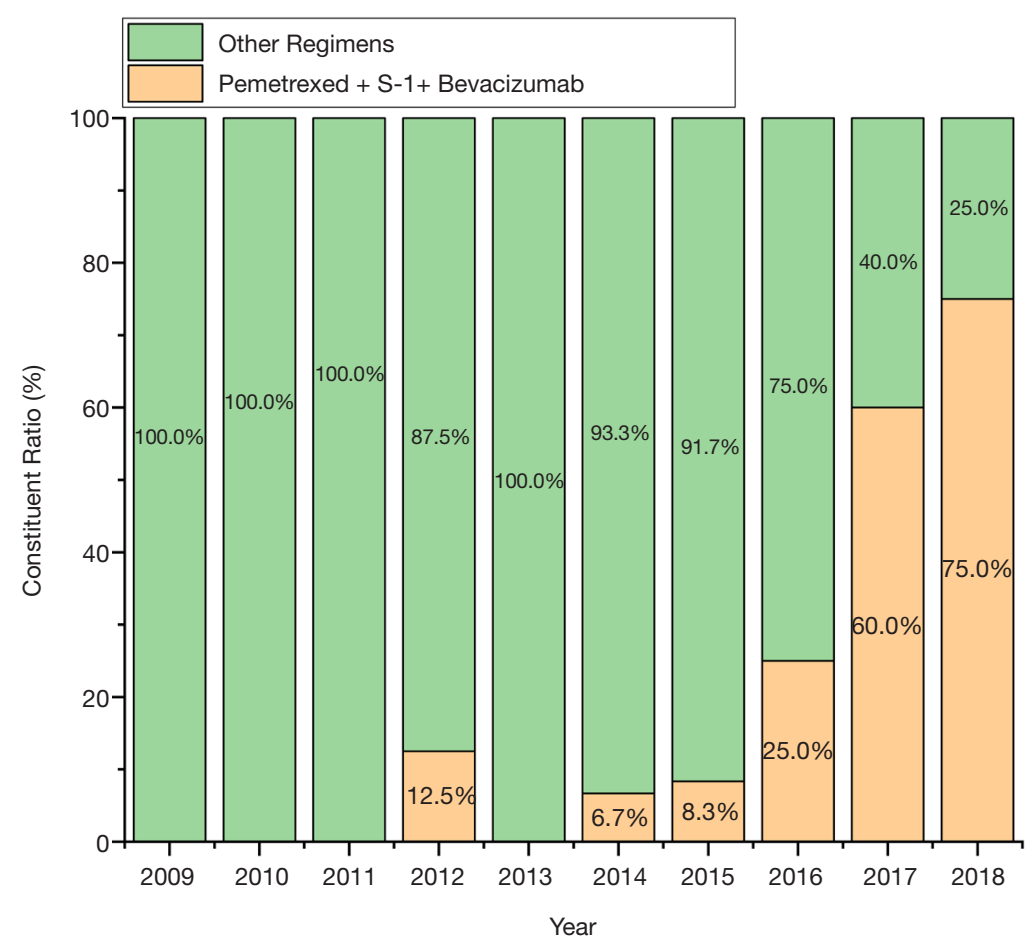

Figure 4 Time distribution of treatment initiation in two groups. The time distribution of treatment initiation in the two groups differed, and the superior treatment group was closer overall.

two types of regulators: gimeracil and oteracil. The main component, tegafur, is a derivative of fluorouracil, which can be activated into fluorouracil through biotransformation in the human body. The basic mechanism of fluorouracil resistance is often accompanied by the up-regulation of dihydropyrimidine dehydrogenase (DPD). Researchers have measured the DPD activity of gastric cancer patients during capecitabine treatment and found there to be a negative correlation between DPD activity and blood concentration (20). Gimeracil, in the S-1 component, was shown to reduce the activity of DPD and increase the sensitivity of patients with DPD activation to fluorouracil drugs (21). Therefore, the use of S-1 as a thirdline treatment for other fluorouracil-resistant tumors is reasonable.

S-1 has also received widespread attention in the treatment of colorectal cancer. In the FIRIS phase III clinical trial, the non-inferiority of S-1 combined with irinotecan compared with the standard FOLFIRI regimen for second-line treatment of metastatic colorectal cancer was demonstrated. However, patients treated with this regimen had a slightly higher incidence of diarrhea (22). A review study published in 2014 described the use of S-1 alone or in combination with other drugs as a first-line, second-line, or adjuvant therapy for colorectal cancer, and also observed that S-1 was associated with a higher incidence of diarrhea (23). As a third-line treatment for colorectal cancer, 41 patients with multiline treatment of colorectal cancer were treated with S-1 and oral tetrahydrofolate in a phase II clinical trial in 2018, reaching an mPFS of 2.55 months with good tolerability (24). Liu et al. also used S-1 monotherapy in 28 patients and achieved an mPFS of 2.5 months (25). Dai et al. used S-1 combined with irinotecan as second-line or third-line treatment to achieve an mPFS of 5.0 months (26). Furthermore, Bu et al. used S-1 monotherapy to obtain an mPFS of 3.3 months in the third-line treatment of elderly patients with metastatic colorectal cancer (27). In 2013 in Lancet Oncology, S-1 was evaluated as an alternative drug for colorectal cancer (28). Therefore, S-1 is an effective chemotherapy drug for the treatment of colorectal cancer.

Aside from the chemotherapeutic drugs in the compatibility of the superior regimen, bevacizumab has also demonstrated treatment efficacy. Bevacizumab is a macromolecular humanized monoclonal antibody that acts on the vascular endothelial growth factor (VEGF). With antitumor angiogenesis effects, it is a widely used 
targeted therapeutic drug. Bevacizumab is considered to be an important drug in the treatment of colorectal cancer. In large clinical trials such as EAGLE, ARTIST, and PEAK, this drug has been shown to be effective and safe in combination with various drugs such as oxaliplatin, irinotecan, and fluorouracil (29-31). Bevacizumab has a strong universality in the treatment of colorectal cancer, and it is not necessary to distinguish the tumor site or KRAS, NRAS, BRAF, and other genomic signatures in patients prior to treatment. Therefore, even if the rate of KRAS mutations in the pemetrexed + S-1 + bevacizumab treatment group was as high as $42.86 \%$, patients could still benefit from the treatment. In our study, bevacizumab had been used more frequently in previous treatments (66.67\%) in the superior regimen group than in the other regimens group, but patients continued to benefit from this drug. In the ML18147 study and the BEBYP study, patients who had previously been treated with bevacizumab were reported to still be able to benefit from using bevacizumab again in later treatment. However, in the ML18147 trial, patients with rapidly progressing tumors (within 3 months) on the previous treatment were not included $(32,33)$. Therefore, bevacizumab as a later treatment could also be a potential therapeutic approach.

Another difference between the two groups was that the treatment period of the superior group was relatively short, which might be closely related to the popularity of bevacizumab and changes in medical insurance policies.

In the study of third-line treatments for colorectal cancer, the development of small molecule-targeted drugs is compelling. However, few breakthroughs in new chemotherapy regimens have been made in this field. Based on a retrospective analysis of the cases in this study, we found a greater therapeutic effect with pemetrexed + S-1 + bevacizumab as a third-line or later treatment of patients with advanced colorectal cancer than with other pemetrexed-based chemotherapy regimens. In combination with a review of previous literature and an evaluation of drug interactions, the program was considered to be worthy of further prospective and exploratory clinical trials to obtain a more accurate assessment of efficacy and toxicity.

This study has several limitations. The total sample size was small, with only 88 evaluable patients, which might have introduced bias, and made it difficult to perform subgroup analyses. Some cases were excluded due to there being no follow-up at 3 months. These patients might have had relatively poor outcomes but were not analyzed, which also could have caused bias. Furthermore, the time span of patient visits was large, and the time span of the target regimens reached up to 9 years. This study also had a large loss to follow-up in the OS data [superior group: 2/21 (9.5\%), other groups: 23/67 (34.3\%), total (28.4\%)]. For some patients, especially patients in the superior group, OS was not reached [superior group: 6/21 (28.6\%), other groups: $4 / 67(6.0 \%)$ ], meaning the estimate for OS might not have been accurate.

\section{Conclusions}

In conclusion, pemetrexed-based chemotherapy was found to be effective and safe for refractory colorectal cancer. Pemetrexed $+\mathrm{S}-1+$ bevacizumab was the optimal regimen for refractory colorectal cancer out of all of the pemetrexedbased treatments assessed. Although this regimen is not guideline-recommended, we still believe it to be an effective treatment approach that warrants further investigation in future clinical trials.

\section{Acknowledgments}

Funding: This work was supported by the National Natural Science Foundation of China (No. 81572389 and 81871944), and the Jiangsu Province Key Medical Talents (No. ZDRCA2016026).

\section{Footnote}

Reporting Checklist: The authors have completed the STROBE reporting checklist Available at http://dx.doi. org/10.21037/atm-20-1095

Data Sharing Statement: Available at http://dx.doi. org/10.21037/atm-20-1095

Conflicts of Interest: All authors have completed the ICMJE uniform disclosure form (available at http://dx.doi. org/10.21037/atm-20-1095). The authors have no conflicts of interest to declare.

Ethical Statement: The authors are accountable for all aspects of the work in ensuring that questions related to the accuracy or integrity of any part of the work are appropriately investigated and resolved. The study was conducted in accordance with the Declaration of Helsinki (as is revised in 2013). The study was approved by the Ethics Committee of the First Affiliated Hospital of Nanjing 
Medical University (2019-SR-301) and individual consent for this retrospective analysis was waived.

Open Access Statement: This is an Open Access article distributed in accordance with the Creative Commons Attribution-NonCommercial-NoDerivs 4.0 International License (CC BY-NC-ND 4.0), which permits the noncommercial replication and distribution of the article with the strict proviso that no changes or edits are made and the original work is properly cited (including links to both the formal publication through the relevant DOI and the license). See: https://creativecommons.org/licenses/by-nc-nd/4.0/.

\section{References}

1. Bray F, Ferlay J, Soerjomataram I, et al. Global cancer statistics 2018: GLOBOCAN estimates of incidence and mortality worldwide for 36 cancers in 185 countries. CA Cancer J Clin 2018;68:394-424.

2. Sun KX, Zheng RS, Zhang SW, et al. Report of Cancer Incidence and Mortality in Different Areas of China. China Cancer 2019;28:1-11.

3. National Comprehensive Cancer Network Guidelines, Colon Cancer, Version 4.2018. Available online: http: // www.nccn.org, accessed October 19, 2018.

4. Fukuoka S, Hara H, Takahashi N, et al. Regorafenib Plus Nivolumab in Patients With Advanced Gastric or Colorectal Cancer: An Open-Label, Dose-Escalation, and Dose-Expansion Phase Ib Trial (REGONIVO, EPOC1603). J Clin Oncol 2020;38:2053-61.

5. Meric-Bernstam F, Hurwitz H, Raghav K, et al. Pertuzumab plus trastuzumab for HER2-amplified metastatic colorectal cancer (MyPathway): an updated report from a multicentre, open-label, phase $2 \mathrm{a}$, multiple basket study. Lancet Oncol 2019;20:518-30.

6. Hong DS, DuBois SG, Kummar S, et al. Larotrectinib in patients with TRK fusion-positive solid tumours: a pooled analysis of three phase $1 / 2$ clinical trials. Lancet Oncol 2020;21:531-40.

7. ALIMTA (pemetrexed disodium) Injection. [cited 2018 Oct 10]. Available online: https://www.accessdata.fda.gov/ drugsatfda

8. Rossi G, Alama A, Genova C, et al. The evolving role of pemetrexed disodium for the treatment of non-small cell lung cancer. Expert Opin Pharmacother 2018;19:1969-76.

9. Zhang G, Xie X, Liu T, et al. Effects of pemetrexed, gefitinib, and their combination on human colorectal cancer cells. Cancer Chemother Pharmacol 2013;72:767-75.
10. Underhill C, Goldstein D, Gorbounova VA, et al. A randomized phase II trial of pemetrexed plus irinotecan (ALIRI) versus leucovorin-modulated 5-FU plus irinotecan (FOLFIRI) in first-line treatment of locally advanced or metastatic colorectal cancer. Oncology 2007;73:9-20.

11. Wu XY, Huang XE, You SX, et al. Phase II study of pemetrexed as second or third line combined chemotherapy in patients with colorectal cancer. Asian Pac J Cancer Prev 2013;14:2019-22.

12. Passardi A, Fanini F, Turci L, et al. Prolonged Pemetrexed Infusion Plus Gemcitabine in Refractory Metastatic Colorectal Cancer: Preclinical Rationale and Phase II Study Results. Oncologist 2017;22:886-e79.

13. Lim SW, Lee S, Lee J, et al. Pemetrexed Monotherapy as Salvage Treatment in Patients with Metastatic Colorectal Cancer Refractory to Standard Chemotherapy: A Phase II Single-arm Prospective Trial. J Cancer 2018;9:2910-5.

14. Zhang W, Cui CX, Liu JB, et al. Evaluation of pemetrexed in the treatment of advanced gastrointestinal malignancies. Chinese General Practice 2015:2042-5.

15. Li J, Qin S, Xu R, et al. Regorafenib plus best supportive care versus placebo plus best supportive care in Asian patients with previously treated metastatic colorectal cancer (CONCUR): a randomised, double-blind, placebocontrolled, phase 3 trial. Lancet Oncol 2015;16:619-29.

16. Li J, Qin S, Xu RH, et al. Effect of Fruquintinib vs Placebo on Overall Survival in Patients With Previously Treated Metastatic Colorectal Cancer: The FRESCO Randomized Clinical Trial. JAMA 2018;319:2486-96.

17. Hurwitz H, Fehrenbacher L, Novotny W, et al. Bevacizumab plus Irinotecan, Fluorouracil, and Leucovorin for Metastatic Colorectal Cancer. N Engl J Med 2004;350:2335-42.

18. Venook AP, Niedzwiecki D, Lenz HJ, et al. Effect of First-Line Chemotherapy Combined With Cetuximab or Bevacizumab on Overall Survival in Patients With KRAS Wild-Type Advanced or Metastatic Colorectal Cancer: A Randomized Clinical Trial. JAMA 2017;317:2392-401.

19. Woods B, Paracha N, Scott DA, et al. Raltitrexed plus cisplatin is cost-effective compared with pemetrexed plus cisplatin in patients with malignant pleural mesothelioma. Lung Cancer 2012;75:261-7.

20. Peng RJ, Dong QM, Shi YX, et al. Correlative Analysis between Serum Dihydropyrimidine Dehydrogenase Activity, Concentration of 5-Fluorouracil and Adverse Events in the Treatment of Advanced Gastric Cancer Patients. Ai Zheng. 2006;25:1039-43.

21. Usuki H, Ishimura K, Yachida S, et al. Dihydropyrimidine 
dehydrogenase (DPD) activity in gastric cancer tissue and effect of DPD inhibitory fluoropyrimidines. Gastric Cancer 2003;6 Suppl 1:66-70.

22. Yasui H, Muro K, Shimada Y, et al. A phase 3 noninferiority study of 5-FU/1-leucovorin/irinotecan (FOLFIRI) versus irinotecan/S-1 (IRIS) as second-line chemotherapy for metastatic colorectal cancer: updated results of the FIRIS study. J Cancer Res Clin Oncol 2015;141:153-60.

23. Miyamoto Y, Sakamoto Y, Yoshida N, et al. Efficacy of S-1 in colorectal cancer. Expert Opin Pharmacother 2014;15:1761-70.

24. Hsu HC, Chou WC, Kuan FC, et al. A Phase II study of S-1 plus oral leucovorin in heavily treated metastatic colorectal cancer patients. Cancer Manag Res 2018;10:6061-70.

25. Liu DL, Wang YH, Lu S, et al. Clinical observation of Tiggio in third-line treatment of advanced colorectal cancer. The Practical Journal of Cancer 2016;31:1564-5.

26. Dai YD, Zhang DX, Tan Y, et al. Clinical observation of irinotecan combined with tiggio second- and thirdline treatment of advanced colorectal cancer. Journal of Practical Oncology 2014;29:186-91.

27. Bu LJ, Wu J, Da J, et al. Clinical Observation of Tiggio Capsules in the Treatment of Elderly Advanced Colorectal Cancer. Anhui Medical Journal 2012;33:416-8.

Cite this article as: Yu Z, Wang J, Cai X, Gao Z, Wang S, $\mathrm{Gu}$ Y. Analysis of pemetrexed-based chemotherapy in the treatment of advanced colorectal cancer. Ann Transl Med 2020;8(21):1368. doi: 10.21037/atm-20-1095
28. Benson AB. S-1: another oral agent for patients with colorectal cancer. Lancet Oncol 2013;14:1244-5.

29. Guan ZZ, Xu JM, Luo RC, et al. Efficacy and safety of bevacizumab plus chemotherapy in Chinese patients with metastatic colorectal cancer: a randomized phase III ARTIST trial. Chin J Cancer 2011;30:682-9.

30. Iwamoto S, Takahashi T, Tamagawa H, et al. FOLFIRI plus bevacizumab as second-line therapy in patients with metastatic colorectal cancer after first-line bevacizumab plus oxaliplatin-based therapy: the randomized phase III EAGLE study. Ann Oncol 2015;26:1427-33.

31. Schwartzberg LS, Rivera F, Karthaus M, et al. PEAK: a randomized, multicenter phase II study of panitumumab plus modified fluorouracil, leucovorin, and oxaliplatin (mFOLFOX6) or bevacizumab plus mFOLFOX6 in patients with previously untreated, unresectable, wild-type KRAS exon 2 metastatic colorectal cancer. J Clin Oncol 2014;32:2240-7.

32. Bennouna J, Sastre J, Arnold D, et al. Continuation of bevacizumab after first progression in metastatic colorectal cancer (ML18147):a randomised phase 3 trial. Lancet Oncol 2013;14:29-37.

33. Masi G, Salvatore L, Boni L, et al. Continuation or reintroduction of bevacizumab beyond progression to firstline therapy in metastatic colorectal cancer: final results of the randomized BEBYP trial. Ann Oncol 2015;26:724-30. 